\title{
Laminar starting plumes in high-Prandtl-number fluids
}

\author{
By EDOUARD KAMINSKI AND CLAUDE JAUPART \\ Institut de Physique du Globe de Paris, Université Paris 7 - Denis Diderot, Paris, France
}

(Received 25 April 2002 and in revised form 1 August 2002)

Experimental studies of laminar axisymmetric starting plumes are performed to investigate the dependence of the flow on the Prandtl number, focusing on large Prandtl numbers. Thermal plumes are generated by a small electric heater in a glass tank filled with viscous oils. Prandtl numbers in the range of $7-10^{4}$ were investigated. Experimental conditions are such that viscosity variations due to temperature differences are negligible. Plumes ascend in two different regimes as a function of distance to source. At short distances, the plumes accelerate owing to the development of the viscous boundary layer. At distances larger than about five times the heater size, the ascent velocity is constant and increases as a function of the Prandtl number, as predicted by theory for steady plumes. This velocity is, within experimental error, proportional to the steady plume centreline velocity.

\section{Introduction}

Thermal convection phenomena play a key role in many natural systems such as the atmosphere, the ocean, magma chambers and the Earth's mantle. At one end of the spectrum, geological flows involve fluids with very large Prandtl numbers (larger than $10^{3}$ for magmas and at least $10^{23}$ for the Earth's mantle) and are routinely studied in the limit of infinite Prandtl number. In this limit, inertial effects are neglected, but the validity of this approximation has not been thoroughly tested for laminar thermal plumes.

Theory for steady laminar plumes is well-established. Scaling arguments indicate that the vertical velocity is constant (Batchelor 1954). Numerical results are available up to a Prandtl number of 10 (e.g. Fujii 1963; Brand \& Lahey 1967; Worster 1986) and an asymptotic analysis for large Prandtl numbers may be found in Worster (1986). These analyses are valid for the plume stem far from the leading edge (the cap) and do not specify the cap behaviour. How the ascent velocity of a plume cap compares with that of the steady stem below is not known. As regards starting plumes, existing laboratory studies (Shlien 1976; Moses, Zocchi \& Libchaber 1993) do not allow an assessment of the Prandtl number dependence. Coulliette \& Loper (1995) found differences between their numerical calculations for very large, but finite, Prandtl number and those of Olson, Schubert \& Anderson (1993) at infinite Prandtl number. One consequence is that it may not be possible to make quantitative comparisons between numerical models for infinite Prandtl number and laboratory experiments.

Our initial motivation for the present work was to study various aspects of mantle plume dynamics in the laboratory. In a viscous oil with a Prandtl number of $10^{3}$, we found that starting plumes were much faster than allowed by the scaling law of Moses et al. (1993). This raised the question of how to extrapolate experimental results to 
geological systems. The type of problem facing geologists is perhaps best explained by the following brief account, which also explains why starting plumes are of special interest. Flood basalts, rare and extremely voluminous volcanic outpourings which punctuate the geological record at intervals of several tens of million years, have been attributed to decompression melting in large mantle plume caps (Campbell \& Griffiths 1990). According to the most popular theory, they are generated by instabilities of a thermal boundary layer at the core-mantle boundary (Stacey \& Loper 1983). Others have argued that they come from a shallower interface within a stratified mantle. Given the large uncertainties on mantle properties and on the thermal power (or buoyancy flux) of mantle plumes, discriminating between these competing models requires robust scaling laws. For example, Courtillot \& Besse (1987) have suggested a link between plume initiation at the core-mantle boundary and changes in the frequency of geomagnetic field reversals, implying that the time lag between such magnetic events and the onset of volcanic activity should be close to the rise time of a plume through the Earth's mantle. Proving this right or wrong depends on the sensitivity of the velocity estimate to the thermal power carried by the plume and to mantle properties such as viscosity and expansion coefficient. Other important goals are to account for the volume and peculiar isotopic composition of flood basalts, which depend on the dynamics of mantle plume caps (Farnetani, Legras, \& Tackley 2002).

In this paper, we study starting plumes rising through viscous oils in the laboratory, up to a maximum Prandtl number of $10^{4}$. The experimental results are compared to theoretical and numerical analyses for steady plumes. Special attention is paid to the initial stages of ascent at small distances from the source.

\section{Steady plume versus starting plume}

The basic scaling relationship for the vertical velocity $W$ in a steady plume was first established by Batchelor (1954) as

$$
W \propto\left(\frac{g \alpha Q}{\rho \nu C_{p}}\right)^{1 / 2},
$$

where the variables are defined in table 1 . Using an asymptotic theory for $\sigma \gg 1$ in an unbounded isoviscous fluid, Worster (1986) found that the centreline velocity is

$$
W_{o}=\left(\frac{\ln \epsilon^{-2}}{2 \pi}\right)^{1 / 2}\left(\frac{g \alpha Q}{\rho \nu C_{p}}\right)^{1 / 2},
$$

where $\epsilon$ is the solution of

$$
\epsilon^{4} \ln \epsilon^{-2}=\sigma^{-1} .
$$

Moses et al. (1993) determined experimentally the ascent velocities of starting plumes in several fluids with different Prandtl numbers. They found that all their measurements are consistent with the following scaling relationship

$$
W_{c}=(0.23 \pm 0.05)\left(\frac{g \alpha Q}{\rho \nu C_{p}}\right)^{1 / 2} .
$$

In fact, their data suggest that the proportionality constant in this relationship depends on the Prandtl number, but they did not pursue the matter further.

Comparing (2.2) and (2.4) raises the obvious question of the relation between the velocities of starting and steady plumes. Theory has been developed using a 


Symbol
$\alpha$
$\rho$
$\mu$
$\nu=\mu / \rho$
$\kappa$
$C_{p}$
$\sigma=v / \kappa$
$g$
$Q$
$R$
$a$
$W$
$L$
$R_{h}$
$\delta_{v}$
$\delta_{\kappa}$

\section{Variable}

Expansion coefficient

Density at $20^{\circ} \mathrm{C}$

Viscosity

Kinematic viscosity

Thermal diffusivity

Specific heat

Prandtl number

Acceleration due to gravity

Power input

Cap radius

Stem radius

Ascent velocity

Plume length

Size of the source

Viscous boundary-layer thickness

Thermal boundary-layer thickness
Dimensions

$\mathrm{K}^{-1}$
$\mathrm{~kg} \mathrm{~m}^{-3}$
$\mathrm{Pas}$
$\mathrm{m}^{2} \mathrm{~s}^{-1}$
$\mathrm{~m}^{2} \mathrm{~s}^{-1}$
$\mathrm{~J} \mathrm{~K}^{-1} \mathrm{~kg}^{-1}$
$\mathrm{~m} \mathrm{~s}^{-2}$
$\mathrm{~J} \mathrm{~s}^{-1}$
$\mathrm{~m}$
$\mathrm{~m}$
$\mathrm{~m} \mathrm{~s}$
$\mathrm{~m}$
$\mathrm{~m}$

TABLE 1. Notation.

boundary-layer approximation valid for plumes of large vertical extent. This requires that the viscous boundary layer $\delta_{v}$ around the plume stem scales as $\sqrt{v z / W}$, where $z$ is height, and that the height of the stem, $L$, is much larger than $\delta_{v}$ :

$$
L \gg v\left(\frac{\rho \nu C_{p}}{g \alpha Q}\right)^{1 / 2} .
$$

For a starting plume, we must make sure that the influence of the cap on the flow is negligible. Resistance to motion comes from viscous stresses acting on the cap and on the stem of the starting plume (figure 1). The plume cap can be described as a 'Rankine vortex' (Moses et al. 1993) of radius $R$, and the force acting on it scales with $R^{2} \mu W / R$. If the stem is approximated by a cylinder of radius $a$ and length $L$, the force acting on it scales with $L a \mu W / \delta_{v}$. Assuming $R \sim a$, the ratio of viscous dissipation due to the cap and the stem is

$$
\frac{\Phi_{\text {cap }}}{\Phi_{\text {stem }}} \sim \frac{\delta_{v}}{L}
$$

The condition that $\Phi_{\text {cap }} / \Phi_{\text {stem }} \ll 1$ is equivalent to the previous one (2.5). These arguments show that starting plumes must be studied at sufficiently large distances from their sources.

\section{Laboratory experiments}

\subsection{Experimental methods}

A $45^{3} \mathrm{~cm}^{3}$ glass tank was filled with a Newtonian fluid and a $5 \mathrm{~mm}$ diameter electrical coil was placed on a small plastic stand at the base. Working fluids were water and silicone oils of various viscosities. For these fluids, viscosity is very weakly sensitive to temperature, which is appropriate for the isoviscous scalings derived above. Physical properties for the fluids are given in table 2. All properties save specific heat were determined in our laboratory. For water, the variation of density with temperature 


\begin{tabular}{lccccc}
\multicolumn{1}{c}{ Fluid } & $\rho\left(\mathrm{kg} \mathrm{m}^{-3}\right)$ & $\mu(\mathrm{Pas})$ & $\alpha\left(\mathrm{K}^{-1}\right)$ & $C_{p}\left(\mathrm{~J} \mathrm{~K}^{-1} \mathrm{~kg}^{-1}\right)$ & $\kappa\left(\mathrm{m} \mathrm{s}^{-2}\right)$ \\
Water & 998 & $1.0 \times 10^{-3}$ & $2.2 \times 10^{-4}$ & 4180 & $1.4 \times 10^{-7}$ \\
47V2 & 972 & $1.9 \times 10^{-3}$ & $1.1 \times 10^{-3}$ & 1970 & $6.2 \times 10^{-8}$ \\
47V8 & 972 & $8.2 \times 10^{-3}$ & $1.1 \times 10^{-3}$ & 1900 & $7.1 \times 10^{-8}$ \\
47V30 & 971 & $2.8 \times 10^{-2}$ & $1.1 \times 10^{-3}$ & 1630 & $9.7 \times 10^{-8}$ \\
47V100 & 973 & $1.1 \times 10^{-1}$ & $1.0 \times 10^{-3}$ & 1630 & $1.0 \times 10^{-7}$ \\
47V500 & 973 & $5.4 \times 10^{-1}$ & $1.0 \times 10^{-3}$ & 1460 & $1.1 \times 10^{-7}$ \\
$47 \mathrm{~V} 10^{3}$ & 974 & 1.1 & $9.5 \times 10^{-4}$ & 1460 & $1.1 \times 10^{-7}$
\end{tabular}

TABLE 2. Physical properties of the experimental fluids. $47 \mathrm{~V} \#$ are the trade names of the different Rhodorsil ${ }^{\mathrm{TM}}$ silicone oils.

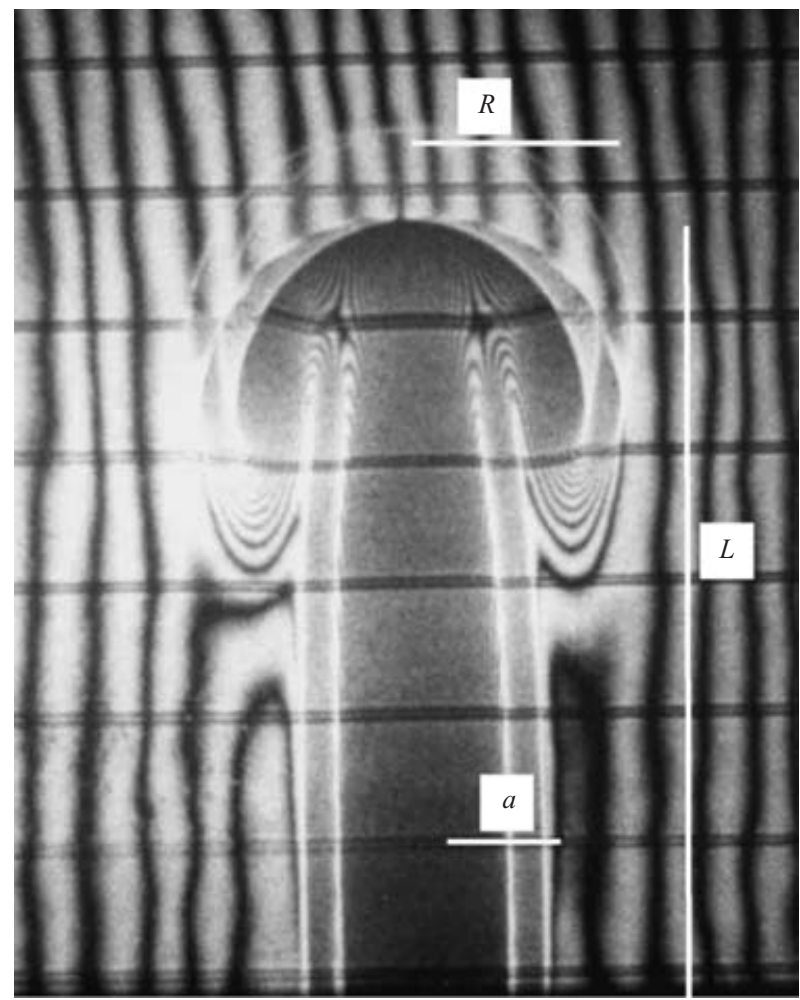

FIGURE 1. Photograph of a starting laminar thermal plume $\left(\sigma=5 \times 10^{3}, Q=2.2 \mathrm{~W}\right)$ using differential interferometry. Extinction lines follow constant horizontal temperature gradients. Vertical extinction lines away from the plume correspond to light beams through uniform background.

is nonlinear and we took the value of the expansion coefficient at room temperature $\left(20^{\circ} \mathrm{C}\right)$. With increasing power input, plume temperatures increase, implying a viscosity contrast with respect to fluid in the far field. To minimize such viscosity variations, we used small values of power input, in a range of $0.01-5 \mathrm{~W}$, which are close to those of Moses et al. (1993). Viscosity differences away from the heater, where we test the scaling laws, never reached a factor of 2 . We took care to 


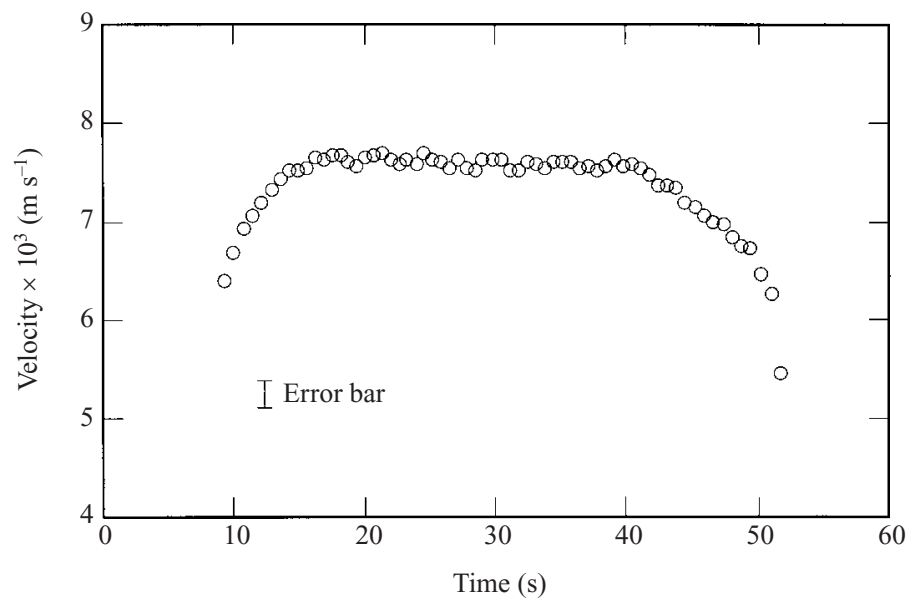

FIGURE 2. Ascent velocity as a function of height for $\sigma=300$ and $Q=1.25 \mathrm{~W}$. This velocity is constant, as predicted by scaling arguments, except at the beginning and end of an experiment. The measurement error is about $\pm 3 \%$.

study how plume velocity depends on power input and to verify that the data agree with the isoviscous scaling law.

\subsection{Velocity measurements}

To visualize the temperature field, we used differential interferometry (Hauf \& Grigull 1970, p. 193) which leads to a very precise image of temperature gradients (figure 1). We measured the times at which the plume head reaches specific heights in the tank. These heights were $0.5 \mathrm{~cm}$ apart and marked on vertical scales pasted on two opposite tank walls. The greatest difficulty was to avoid parallax effects. To achieve maximum accuracy, we placed the tank on a moving table whose vertical position was continuously adjusted during an experiment. In this way, the optical and viewing systems were the same at all plume heights. We made sure that the movement of the tank was sufficiently slow to avoid inertial effects.

For each experiment, the raw data are the arrival times of the plume cap at each height. These appear to increase linearly; however, a single linear regression through the whole data set may lead to an inaccurate result. Once the heater is switched on, there is no motion for some time, and hence there is an initial transient with gradually increasing velocity. A similar problem is encountered at large times when the plume slows down as it approaches the top of the fluid. A regression through all data points would include both effects, and hence would lead to an average velocity value smaller than the peak value. To avoid this, we measured local velocity values using a linear regression through the arrival times at five successive heights (figure 2).

For comparisons with theory for an infinite volume of fluid, three experimental artefacts must be overcome: sidewall effects, free-surface effects and thermal power leakage. All these effects act to lower the plume velocity. Additionally, the finite size of the plume source affects the development of the thermal and viscous boundary layers at small heights (Moses et al. 1993). Thus, the plume must be sufficiently far from the source to be in the boundary-layer regime and to be unaffected by the source size. In practice, for $\sigma \leqslant 5 \times 10^{3}$, the former condition, (2.5), is fulfilled at small heights (less than a few centimetres), whereas the latter requires larger distances. For $\sigma=10^{4}$, the latter condition is achieved first. The source effect will be studied in detail below. 


\subsection{Wall effects and free-surface effects}

In each experiment, the ascent velocity decreases at the end. Depending on the experimental conditions, this decrease may be due to wall effects, free-surface effects or a combination of both. For the sake of accuracy, we did not try to correct for wall and free-surface effects using equations derived for simple shapes. For each fluid, wall effects were detected by performing a reference experiment twice at the largest power input (corresponding to the largest plume): first in the $45^{3} \mathrm{~cm}^{3}$ tank, and then in a smaller one $\left(30^{3} \mathrm{~cm}^{3}\right)$. For Prandtl numbers up to $5 \times 10^{3}$, the plateau velocity was found to be the same in the two experiments. For $\sigma=10^{4}$, there was a slight difference between the two, but it was within the error range and hence we kept the results obtained in the large tank.

To avoid wall effects, the viscous boundary layer $\delta_{v}$ must not extend to the tank walls. For tank half width $R_{\text {tank }}$, this states that:

$$
L<\frac{R_{\text {tank }}^{2}}{\nu}\left(\frac{g \alpha Q}{\rho \nu C_{p}}\right)^{1 / 2} .
$$

This condition can be combined with the previous condition, (2.5), to obtain the maximum Prandtl number for which the boundary-layer solution can be observed,

$$
\sigma_{\max } \approx\left(\frac{R_{\text {tank }}^{2} g \alpha Q}{\rho C_{p} \kappa^{3}}\right)^{1 / 3}
$$

For our experimental conditions, the maximum Prandtl number is $\sigma_{\max }=10^{4}$, which is consistent with the observations.

\subsection{The energy flux into the plume}

Heat was generated by ohmic dissipation in a small coil $(5 \mathrm{~mm}$ diameter, resistance of $13 \Omega$ ). We repeated a few experiments with a larger coil dimension $(10 \mathrm{~mm}$ diameter, $18 \Omega$ ) and found no detectable effect on the value of the peak velocity. One problem is that the total power dissipated in the electric coil may not be carried into the plume. We observed a small region of heated fluid below the coil. In steady-state conditions, therefore, some power was diverted towards the small stand at the base to maintain such a heated region. The plume energy flux must therefore be smaller than the total power dissipated in the coil. The latter is measured exactly, but the former is difficult to determine a priori. We took this into account when treating the data, as explained below. Failure to account for this inevitable effect leads to an overestimate of the true energy flux of the plume, and hence, once again, to an underestimate of the proportionality constant in the velocity scaling law.

\section{Experimental results}

\subsection{Ascent in the experimental tank}

By definition, we measure the velocity with which the thermal plume propagates, that is, we measure the advection velocity of the temperature field. It appears that velocity is effectively constant during a large part of the experiment, as predicted by scaling arguments. Four different ascent stages may be defined. The first stage is the conductive growth of a thermal boundary layer around the heater. Bulk motion begins after a finite time which is a decreasing function of the power input and an increasing function of fluid viscosity, as already described by Moses et al. (1993). In the second stage, the cap velocity rapidly increases until it reaches a constant value. 


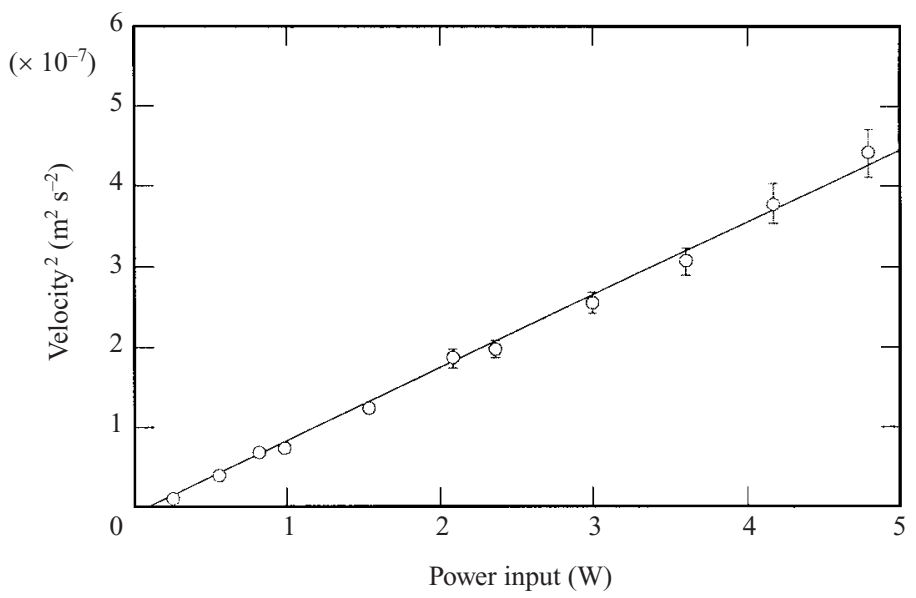

FIGURE 3. The plume rise velocity as a function of power input for $\sigma=10^{4}$. Points are shown with the $3 \%$ experimental error. The solid line corresponds to the linear regression.

In the third stage, velocity remains constant. We shall refer to this as the 'plateau' stage (illustrated figure 1). Later in the experiment, velocity begins to decrease owing to the free-surface effect. In the 'plateau' stage, the cap radius gradually increases following the behaviour already described by Moses et al. (1993).

\subsection{The 'plateau' stage}

In each experiment, this regime is such that velocity is constant within the experimental error, which is about 3\% (mainly visual inaccuracy). The scaling developed for steady plumes predicts that, for a given fluid, velocity increases as $\sqrt{Q}$. This behaviour is shown for oil $47 \mathrm{~V} 10^{3}\left(\sigma=10^{4}\right)$ for which we used the largest range of power inputs (figure 3). Values for $W_{c}^{2}$ and $Q$ lie along a straight line, but the best-fit line does not go through the origin. We interpret this as being due to the downward transport of energy towards the base of the tank, as discussed above. This implies that the power carried by the plume is smaller than the total dissipation in the electric coil. The data are consistent with a relationship of the form:

$$
W_{c}=\beta\left(Q-Q_{o}\right)^{1 / 2},
$$

where $Q_{o}$ is the power 'leakage'. This suggests that this leakage is independent of the total power dissipated. Power leakage increases as the fluid viscosity increases, which may be explained as follows. In the initial stage before flow starts, heating propagates radially away from the coil. The 'lift-off' time increases with increasing fluid viscosity (Moses et al. 1993) and hence the size of the heated region below the electric coil increases with increasing fluid viscosity.

For each fluid, we have measured a constant ascent velocity, and we have written it as follows:

$$
W_{c}=\lambda(\sigma)\left(\frac{g \alpha\left[Q-Q_{o}\right]}{\rho \nu C_{p}}\right)^{1 / 2} .
$$

Parameters $\lambda$ and $\left(Q-Q_{o}\right)$ in equation (4.1) are the relevant experimental results for comparison with theory (table 3 ). For fluids with viscosities smaller than $2.8 \times$ $10^{-2} \mathrm{Pas}\left(\sigma \leqslant 3.0 \times 10^{2}\right)$, power leakage is negligible and the data do demonstrate that velocity is proportional to the square root of power input. At larger viscosity, 
Experimental fluid

Water
$47 \mathrm{~V} 2$
$47 \mathrm{~V} 8$
$47 \mathrm{~V} 30$
$47 \mathrm{~V} 100$
$47 \mathrm{~V} 500$
$47 \mathrm{~V} 10^{3}$

Prandtl number, $\sigma$

$$
7.16
$$$$
31.5
$$$$
119
$$$$
297
$$

$1.13 \times 10^{3}$

$5.05 \times 10^{3}$

$1.03 \times 10^{4}$

Dimensionless velocity, $\lambda$ Power leakage, $Q_{o}(\mathrm{~W})$

$$
\begin{aligned}
& 0.22 \pm 0.02 \\
& 0.31 \pm 0.02 \\
& 0.41 \pm 0.02 \\
& 0.44 \pm 0.02 \\
& 0.46 \pm 0.02 \\
& 0.51 \pm 0.02 \\
& 0.53 \pm 0.02
\end{aligned}
$$

$$
\begin{aligned}
& 2.4 \times 10^{-6} \\
& 1.1 \times 10^{-5} \\
& 2.2 \times 10^{-3} \\
& 8.7 \times 10^{-3} \\
& 1.0 \times 10^{-2} \\
& 4.0 \times 10^{-2} \\
& 6.0 \times 10^{-2}
\end{aligned}
$$

TABLE 3. Experimental results for starting plumes.

the data at small values of the power input must be corrected for power leakage and the demonstration may seem less convincing. However, at large values of the power input $(\geqslant 1 \mathrm{~W})$, power leakage is a small fraction of the total, and velocity is nearly proportional to $Q^{1 / 2}$. We thus conclude that the scaling law is consistent with the experimental data.

For water, our experimental determination for $\lambda$ is $0.22 \pm 0.02$, which is nearly identical to the value reported by Moses et al. (1993). For larger values of the Prandtl number, however, our results are systematically larger than theirs.

\subsection{Dependence on Prandtl number}

According to our experiments, the starting plume velocity increases as a function of the Prandtl number (table 3) and hence it is logical to test whether it is proportional to the stem velocity. For comparison with our measurements, we have obtained new solutions to the steady plume governing equations numerically. We used the same boundary-layer approximation and the same equations as Fujii (1963) and Worster (1986). However, we are dealing with large Prandtl numbers, and hence we have rescaled variables using the thickness of the thermal boundary layer, $\delta_{\kappa}$, instead of the viscous boundary layer. With our scheme, we reproduce the results quoted by Worster (1986) for $\sigma=10$. The ascent of the cap, as measured in our experiments, represents the advection of the thermal anomaly of the plume. An average velocity of heated fluid may be defined as follows

$$
\bar{w}=\frac{\int_{0}^{\infty} w(r) \theta(r) r \mathrm{~d} r}{\int_{0}^{\infty} \theta(r) r \mathrm{~d} r},
$$

where $\theta$ is the temperature anomaly in the plume and $w$ the vertical velocity (for the plumes of this study, both variables are positive).

In figure 4, we compare our numerical results for the centreline velocity to the asymptotic predictions of Worster (1986). The difference between the two decreases with increasing Prandtl number and is less than $6 \%$ for $\sigma \geqslant 10^{2}$. The 'bulk' velocity also tends to the centreline value. This is explained by the fact that, with increasing Prandtl number, the viscous boundary layer becomes increasingly larger than the thermal boundary layer. Thus, for sufficiently large $\sigma$, we have $w \approx W_{o}$ for $0<r<\delta_{\kappa}$. In this limit, $\int_{0}^{\infty} w \theta r \mathrm{~d} r \approx W_{o} \int_{0}^{\infty} \theta r \mathrm{~d} r$, and hence $\bar{w} \approx W_{o}$. To summarize, at large Prandtl number, the two velocities - bulk and centreline - tend to the same value, and to the asymptotic result of Worster (1986). Surprisingly, the 


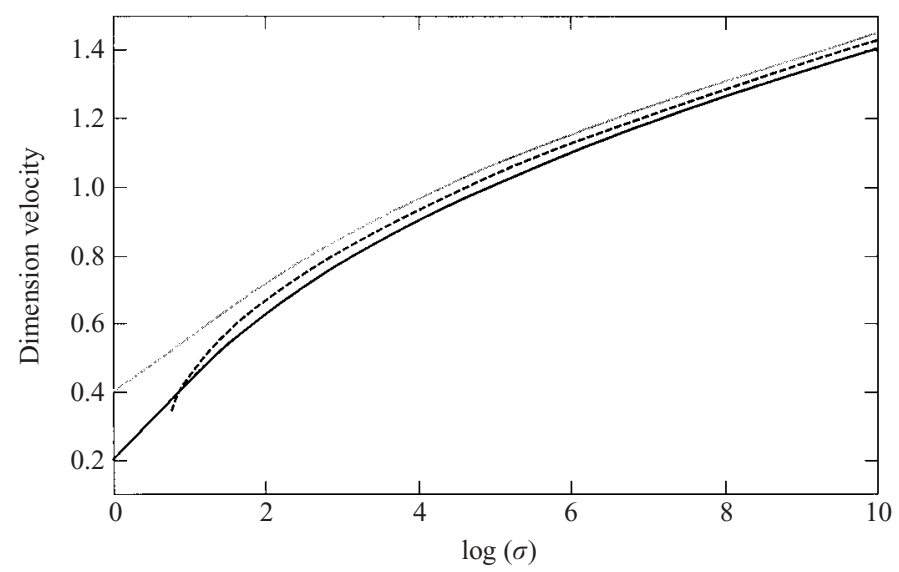

FiguRE 4. Comparison between numerical calculations of the centreline (grey solid line) and bulk (black solid line) velocities of a steady plume and the asymptotic prediction of Worster (1986) (dashed line). Velocity values are scaled with $\sqrt{g \alpha Q / \rho \nu C_{p}}$. The asymptotic prediction is close to both numerical results for $\sigma>10^{2}$.

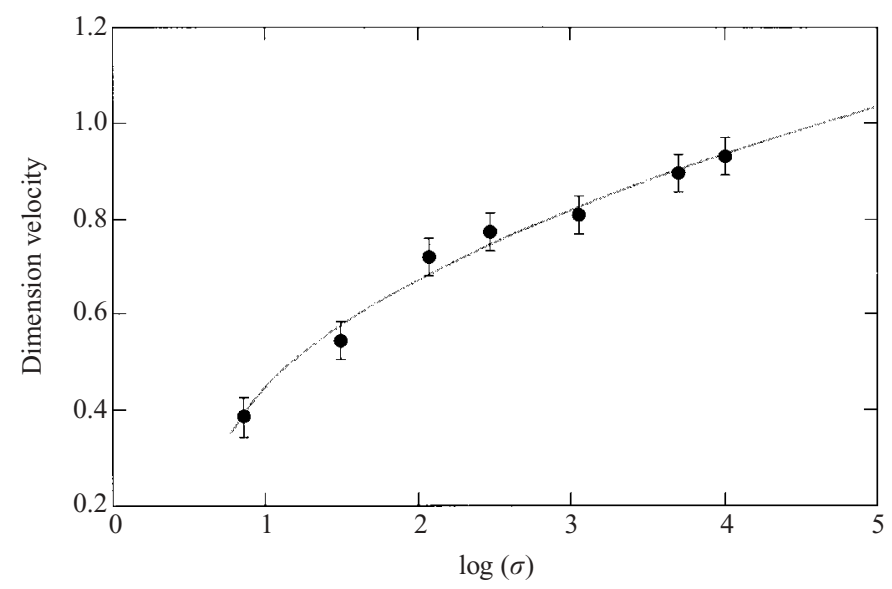

Figure 5. Dimensionless vertical velocity as a function of Prandtl number. @, Present experimental results for starting plumes scaled by a factor of $1 / 0.57$. The solid line shows the asymptotic prediction of Worster (1986) for steady plume stems.

asymptotic result provides a very good approximation for the 'bulk' velocity value for all Prandtl numbers larger than about 7 (the asymptotic equation has no solution for $\sigma<5.47)$.

We found that, within experimental error, our experimental determinations of the cap velocity are proportional to the 'bulk' velocity values for all Prandtl numbers studied. As a consequence, they are also proportional to the asymptotic predictions. This is shown in figure 5, where we plot both the asymptotic results and our experimental values scaled by the best-fitting factor of $1 / 0.57$. For Prandtl numbers larger than about $10^{2}$, the three theoretical velocity values - bulk, centreline and asymptotic - are almost indistinguishable, and hence the experimental data are proportional to all of them within experimental error. 


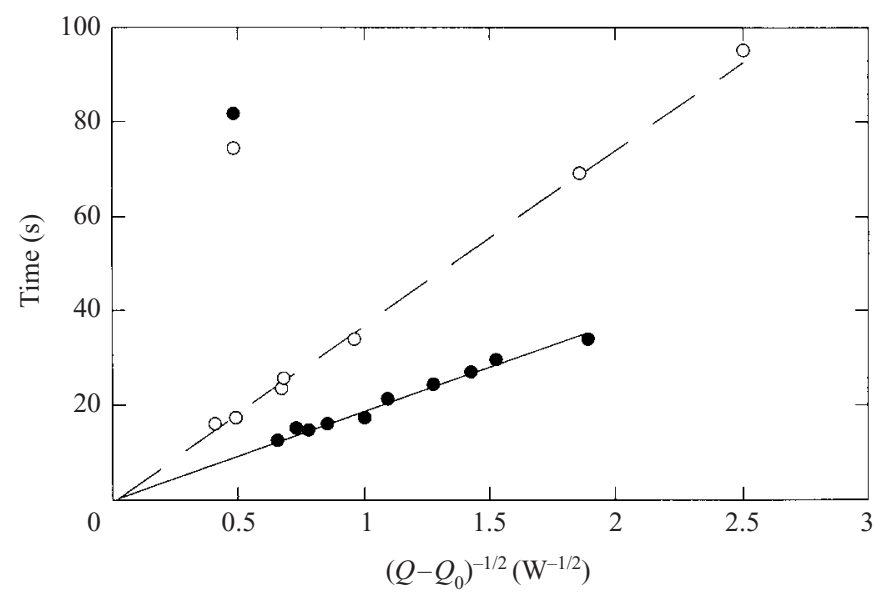

FIGURE 6. Starting time $\tau$ for the plateau regime as a function of the square root of the power input for $\bullet, \sigma=10^{3}$ and $\bigcirc, \sigma=5 \times 10^{3}$. The linear fit is consistent with simple scaling arguments given in the text.

From these results, the ascent velocity of a laminar starting plume can be written as

$$
W_{c}=(0.57 \pm 0.02)\left(\frac{\ln \epsilon^{-2}}{2 \pi}\right)^{1 / 2}\left(\frac{\alpha g Q}{\rho \nu C_{p}}\right)^{1 / 2},
$$

where $\epsilon$ is a root of $\epsilon^{4} \ln \epsilon^{-2}=\sigma^{-1}$ and where $\sigma$ is larger than 7. The starting plume velocity is therefore smaller than velocities in the stem, which implies that the cap is growing with time owing to the input of heated fluid from below.

\subsection{Source effect}

We have studied the transition between the acceleration regime and the plateau regime for intermediate values of the Prandtl number, $\sigma=10^{3}$ and $\sigma=5 \times 10^{3}$. At smaller Prandtl numbers, the acceleration stage is too short to be studied with accuracy. The condition for the plume to reach the plateau regime can be expressed as a function of the size of the heater $R_{h}$,

$$
L \gg R_{h} .
$$

Using $L \sim W t$, this condition can be rewritten as a limit time,

$$
t>A R_{h}\left(\frac{\rho \nu C_{p}}{g \alpha Q}\right)^{1 / 2},
$$

where $A$ is a constant. To test this relationship, we determine time $\tau$ at which the ascent velocity reaches $97 \%$ of the plateau velocity. Figure 6 shows $\tau$ as a function of $\sqrt{Q-Q_{o}}$ for $\sigma=10^{3}$ and $\sigma=5 \times 10^{3}$. The data are consistent with equation (4.6) with $A=18 \pm 1$. This time corresponds to a height above source which is about five times the heater size. We may use $\tau$ as a time scale to obtain a dimensionless relationship $W / W_{c}=f(t / \tau)$. Using these scales, data for 10 experiments in two different oils spanning a large range of power inputs collapse onto a single curve (figure 7). 


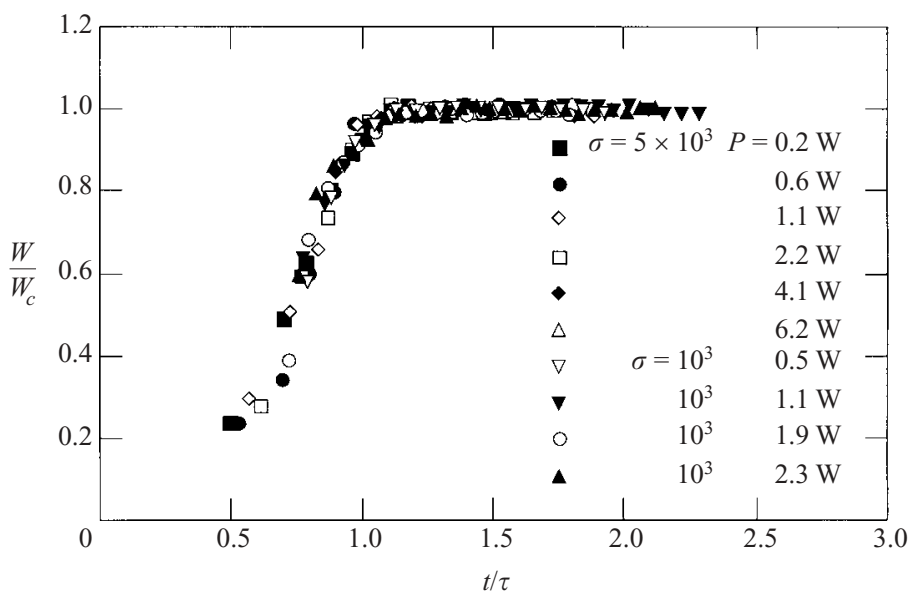

FiguRE 7. Dimensionless ascent velocity as a function of dimensionless time for $\sigma=10^{3}$ and $\sigma=5 \times 10^{3}$. The final velocity decrease due to free-surface effects has been omitted. The plume is in the boundary-layer regime for $t>\tau$.

\section{Conclusion}

Experimental studies in viscous fluids with large Prandtl numbers illustrate that a laminar thermal plume rises in different regimes depending on the height above source. At small height above source, the plume accelerates progressively. At large distance from the source, or equivalently at large times after lift-off, its velocity is constant and proportional to the steady-plume velocity. The starting plume cap grows by continuous addition of fluid from the stem.

The Prandtl-number dependence of the ascent velocity is weak. Geological flows, however, involve fluids with a very large range of physical properties. Applying the scaling law of Moses et al. (1993) to mantle plumes $\left(\sigma \approx 10^{23}\right)$, for example, would lead to a velocity value which would be too small by a factor of 5.7.

The authors thank two anonymous reviewers and Grae Worster for their constructive remarks.

\section{REFERENCES}

Batchelor, G. K. 1954 Heat convection and buoyancy effects in fluids. Q. J. R. Met. Soc. 80, 339-358.

Brand, R. \& Lahey, F. 1967 The heated laminar vertical jet. J. Fluid Mech. 29, 305-315.

CAMpbell, I. \& Griffiths, R. 1990 Implications of mantle plume structure for the evolution of flood basalts. Earth Planet. Sci. Lett. 99, 79-93.

COUlliette, D. \& Loper, D. 1995 Experimental, numerical and analytical models of mantle starting plumes. Phys. Earth Planet. Int. 92, 143-167.

Courtillot, V. \& Besse, J. 1987 Magnetic field reversals, polar wander, and core-mantle coupling. Science 237, 1140-1147.

Farnetani, C., Legras, B. \& TACKLey, P. 2002 Mixing and deformations in mantle plumes. Earth Planet. Sci. Lett. 196, 1-15.

FuJII, T. 1963 Theory of the steady laminar natural convection above a horizontal line source and a point heat source. J. Heat Mass Transfer 16, 597-606.

Hauf, W. \& Grigull, U. 1970 Optical methods in heat transfer. In Advances in Heat Transfer (ed. J. P. Hartnett \& T. F. Irvine Jr), pp. 133-366. Academic. 
Moses, E., Zocchi, G. \& Libchaber, A. 1993 An experimental study of laminar plumes. J. Fluid Mech. 251, 581-601.

Olson, P., Schubert, G. \& Anderson, C. 1993 Structure of axisymmetric plumes. J. Geophys. Res. 98, 6829-6844.

Shlien, D. J. 1976 Some laminar thermal and plume experiments. Phys. Fluids 19, 1089-1098.

StaCEY, F. \& Loper, D. 1983 The thermal boundary layer interpretation of $D^{\prime \prime}$ and its role as a plume source. Phys. Earth Planet. Int. 33, 45-55.

Worster, M. G. 1986 The axisymmetric laminar plume: asymptotic solution for large Prandtl number. Stud. Appl. Maths 75, 139-152. 